\title{
A Model of Creating Hospital Strategic Alliances
}

\author{
Chief Assist. Prof. PhD Boncho Mitev \\ University of Economics - Varna, Varna, Bulgaria \\ bonchomitev@ue-varna.bg
}

\begin{abstract}
Different forms of partnership in the hospital sector exist depending on the degree of integration between the individual participants, the types of partners, their goals and motives for cooperation. The theory describes many conceptual frameworks of the strategic alliance formation process. The purpose of the publication is to analyze some of the existing models and as a result to present a model of creating hospital strategic alliances. This framework integrates the strategic process of cooperation with the particular features of healthcare management. Some of the elements in the model are specific and derive from the characteristics of the Bulgarian healthcare system. The emphasis in its application is the creation of a successful strategic alliance.
\end{abstract}

Keywords: strategic alliances, model of strategic alliance formation, hospital, hospital alliances.

JEL Code: 111,118, MOO

DOI: https://doi.org/10.36997/IJUSV-ESS/2020.9.3.96

\section{Въведение}

Динамичните промени в съвременната бизнес среда превръщат алиансите и останалите форми на коопериране във важен инструмент за реализиране на стратегическите цели на компаниите. Те залагат на партньорските отношения, които се развиват на базата на собственото поле на компетенции на организациите, свързани с отличителните им способности, знания и умения по отношение на определена операция, дейност или процес. Двигател на тяхното създаване е осъзнатата взаимна изгода за участниците, а разнообразните мотиви се свеждат основно до недостига на ресурси или до наличието на неоползотворен капацитет за развитие.

Партньорствата между компаниите в бизнеса имат разнообразни проявления, форми и обхват, и представляват най-общо споразумения между две или повече страни за постигане на общи цели и за защита на общи интереси. В научните среди широко се дискутира въпросьт за стратегическия характер на алиансите. В тази връзка можем да отбележим, че всяко партньорство (сътрудничество, коопериране, съюз, алианс) между организации е стратегическо, когато са изпълнени следните три условия: първо, съвместната дейност да е от стратегическо значение за участниците и тяхното развитие; второ, да съществува стратегическа взаимозависимост между целите на страните в него, и трето, организациите да запазват своята самостоятелност в рамките на партньорството.

Освен в областта на бизнеса, подобно сътрудничество се реализира и в сферата на здравеопазването. Функционирането на лечебните заведения в условия на ресурсна ограниченост и интензивна конкуренция предполага използването на ефективни методи на управление, голяма гъвкавост и умения за работа в динамична среда. Създаването и развиването на партньорски отношения в болничния сектор предоставя възможности за осигуряване на критични за развитието на лечебните заведения ресурси, достъп до нови пазари или технологии. В този смисъл кооперирането може да се използва като инструмент за формиране на конкурентни предимства и за затвърждаване на пазарната им позиция.

В практиката съществуват различни форми на проявление на партньорства в болничния сектор в зависимост от степента на интеграция между отделните участници, видовете партньори, техните цели и мотиви за сътрудничество (Mitev et al., 2006). Най-общо при тях се развиват хоризонтални връзки, когато участниците са само болници, вертикални връзки в случаите на участие на други лечебни заведения, интегрирани в болничното обслужвани или връзки от диверсификационен тип, в които се включват организации, работещи в и/или извън сферата на здравеопазване (Zafirova et al, 2007). Партньорствата се 
реализират в три основни организационни форми - неформално сътрудничество, договорни споразумения и капиталови съюзи, предполагащи сътрудничество със създаване на нова организация или участие в капитала без създаване на нова организация (Lewis, 1990).

В теорията са описани множество концептуални рамки за формиране на стратегически съюз, които са общовалидни за различните му разновидности. Целта на публикацията е да се анализират част от съществуващите модели и да се предложи собствен модел за създаване на стратегически алианси в болничния сектор.

\section{1. Преглед на теоретичните рамки за създаване на стратегически алианси}

Формирането на стратегически алианси се определя като последователен процес и е обект на изследване от редица автори (Ring et al., 1994; Daz et al., 1997; Brouther et al., 1997; Buchel, 2000; Hoffman et al., 2001). Дж. Луиз разграничава четири етапа в този процес: 1) определяне на целите на алианса, 2) избор на типа алианс, 3) анализ на възможностите за създаване на алианс и 4) избор на партньори и формиране на алианса (Lewis, 1990). Кийн и МакДоналд посочват, че инициаторът на стратегическия съюз реализира три последователни дейности: 1) идентифициране на алианса, 2) оценка на възможностите и 3) преговори с потенциалните парньори (Keen et al., 2000). Даз и Тенг дефинират 7 етапа в процеса на тяхното създаването: 1) вземане на решение за формиране на алианс, 2) избор на партньор, 3) договаряне на споразумението, 4) формализиране на алианса, 5) реализиране на съвместната дейност, 6) оценка на изпълнението и 7) промяна.

Анализът показва, че различия между изследователите се открояват главно в броя на етапите и техния обхват. Липсата на съществени противоречия ни дава основание да изведем следните три основни фази в процеса на създаването на стратегически алианси (Mitev, 2005): А) планиране на взаимоотношенията (стратегически анализ и вземане на решение за сьтрудничество, избор на организационна форма на коопериране, избор на партньор, договаряне между страните и сключване на споразумение), Б) реализиране на партньорството (осъществяване на съвместната дейност, управление на ваимоотношенията) и В) оценка на действието на алианса (анализ и оценка на постигнатите резултати, промяна и/или прекратяване на партньорските отношения).

Най-широко застьпен в специализираната литература относно създаването на стратегически алианси е процесния подход, който най-добре е обобщен в два модела - на Мицухаши и на Уипъл и Франкел. Първият модел (фиг.1) представя процеса на формиране на алианс в пет стъпки, който стартира с идентифициране на възможностите за създаване на съюз и завършва със сключване на споразумение между страните.

Моделът на Мицухаши показва формирането на стратегическия алианс като ясен, последователен и не сложен процес, чиято полезност се откроява в начина, по който са описани различните стьпки за реализиране на съюза. Авторът прави разлика между активното и пасивното поведение на компанията в зависимост от нейната роля в този процес - като инициатор или включващ се в алианса. Същевременно обаче, съществуват критики, че това е твърде опростен поглед върху цялостния процес и не се отчитат някои специфични аспекти на партньорските отношения, които биха създали условия на несигурност при осъществяването на отделните етапи в него (Kinderis, 2013).

По-детайлен поглед върху създаването на стратегическите алианси демонстрират Уипъл и Франкел, чийто модел включва идентифициране на необходимостта от алианс, търсене и избор на партньори, управление и оценка на съюза (фиг. 2). Впечатление прави, че авторите разграничават два паралелни потока от дейности, които се осъществяват в хода на процеса - стратегически и оперативен. Те отбелязват, че стратегическият аспект на формирането на алианса (т.е. как той се вписва в общите стратегически цели) не може да бъде отделен от оперативните действия (т.е. необходимостта от свързване на алианса с ежедневните операции). 


\section{IZVESTIA JOURNAL OF THE UNION OF SCIENTISTS - VARNA}

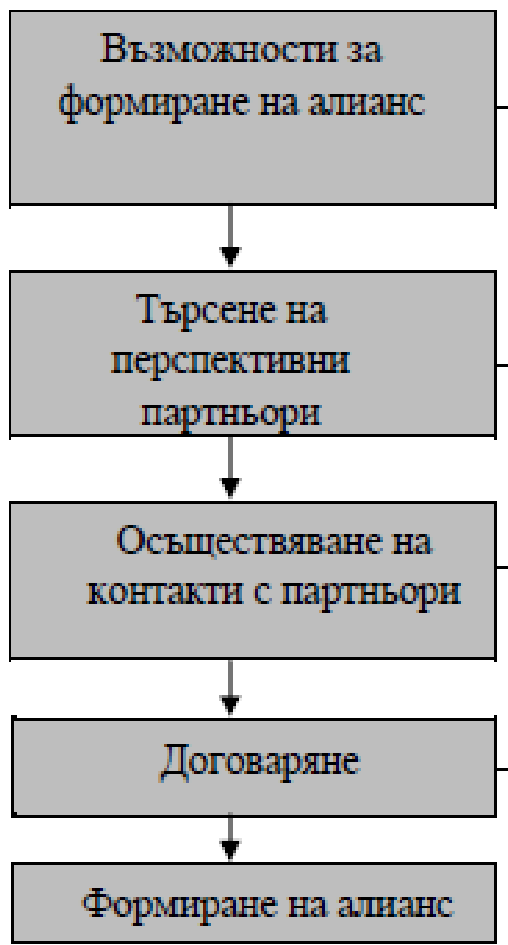

Възможности за формиране на алианс, преглед на бизнес стратегинте, дейностите и компетенциите на партньорите.

Идентифициране на потенциалните партньори и тяхната адекватност към сьществените условия за формиране на алианс.

Осьшествяване на контакти с перспективните потенциални партньори.

Размяна на конфиденциална информация с партньорите. Оценка на компетенциите на партньорите и тяхната надеждност.

Фигура 1. Модел за формиране на алианси (Mitsuhashi, 2002, p.113)

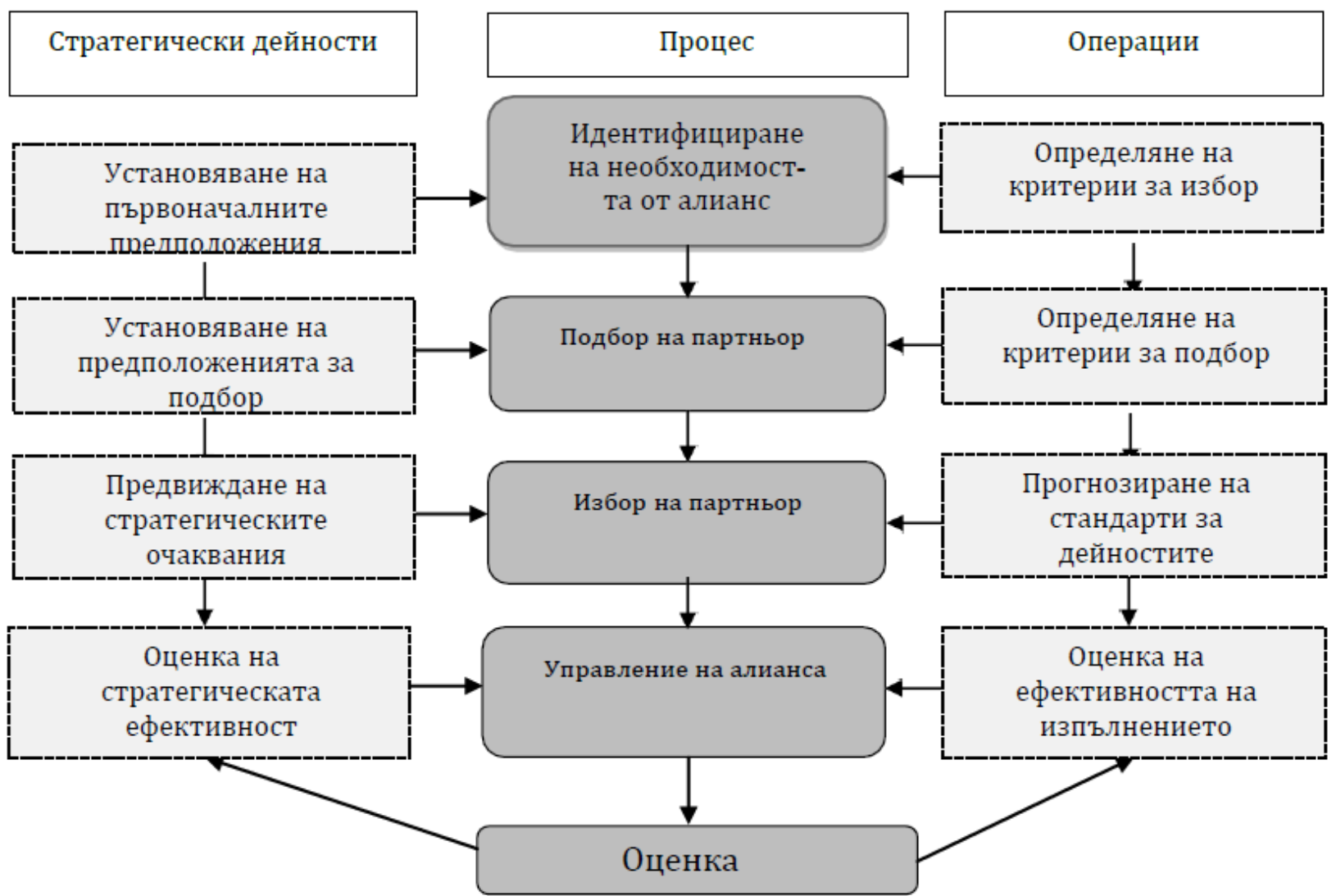

Фигура 2. Модел за формиране на алианси (Whipple and Frankel, 1998, p.338) 
Разглеждайки модела в хорионтален план, Уипъл и Франкел посочват, че процесьт на създаване на алианси всъщност се състои от три стъпки: концептуализация, убеждаване и потвърждаване (Seppälä, 2004). Първата е свързана с промяна в стратегията, която се налага в резултат от действието на конкурентните сили и включва поставяне на целите и критериите за търсене на необходимите характеристики на подходящия партньор. Във втората стьпка организацията усъвършенства своите цели и критерии за подбор, с което се стеснява кръга на потенциалните партньори. Накрая, компанията избира своя партньор и сьвместно вземат решение за формалното създаване на съюз между тях. В третата фаза се разглеждат въпроси, свързани със стратегическата и оперативната ефективност на алианса.

Въпреки че този модел логически свързва създаването на алианса със стратегията на организацията - инициатор, той също търпи критики за подчертано ограничения си поглед върху процеса на формиране на стратегическото партньорство.

Общото между разгледаните модели е, че и двата имат за цел да опишат процеса на създаване на стратегически алианси от гледна точка на последователността от действия, които трябва да са предприемат. По-различен поглед предлага Сепала, чийто модел има за цел да опише начина на мислене и концептуалната рамка на формирането на стратегическия съюз, в опит да обясни техния динамичен характер, който понякога ги превръща в неефективен инструмент за постигане на конкурентни предимства. Този модел представлява алтернативен начин за проследяване на формирането на алианса, който не отрича, а по-скоро допълва пьрвия подход чрез различна гледна точка.

Авторьт изяснява създаването на стратегическия алианс от гледна точка на четири ключови съображения, които предполагат конкретни действия и водят до очаквани резултати (фиг. 3). В началото се осъществява преглед на стратегическото намерение за формиране на съюз, който трябва да потвърди две допускания от съществено значение: първо, че стратегическото намерение наистина е в съответствие със стратегическия контекст на организацията - инициатор, и второ, че резултатьт от стратегическото намерение се разбира и възприема по един и същ начин от хората, участващи в създаването на алианса.

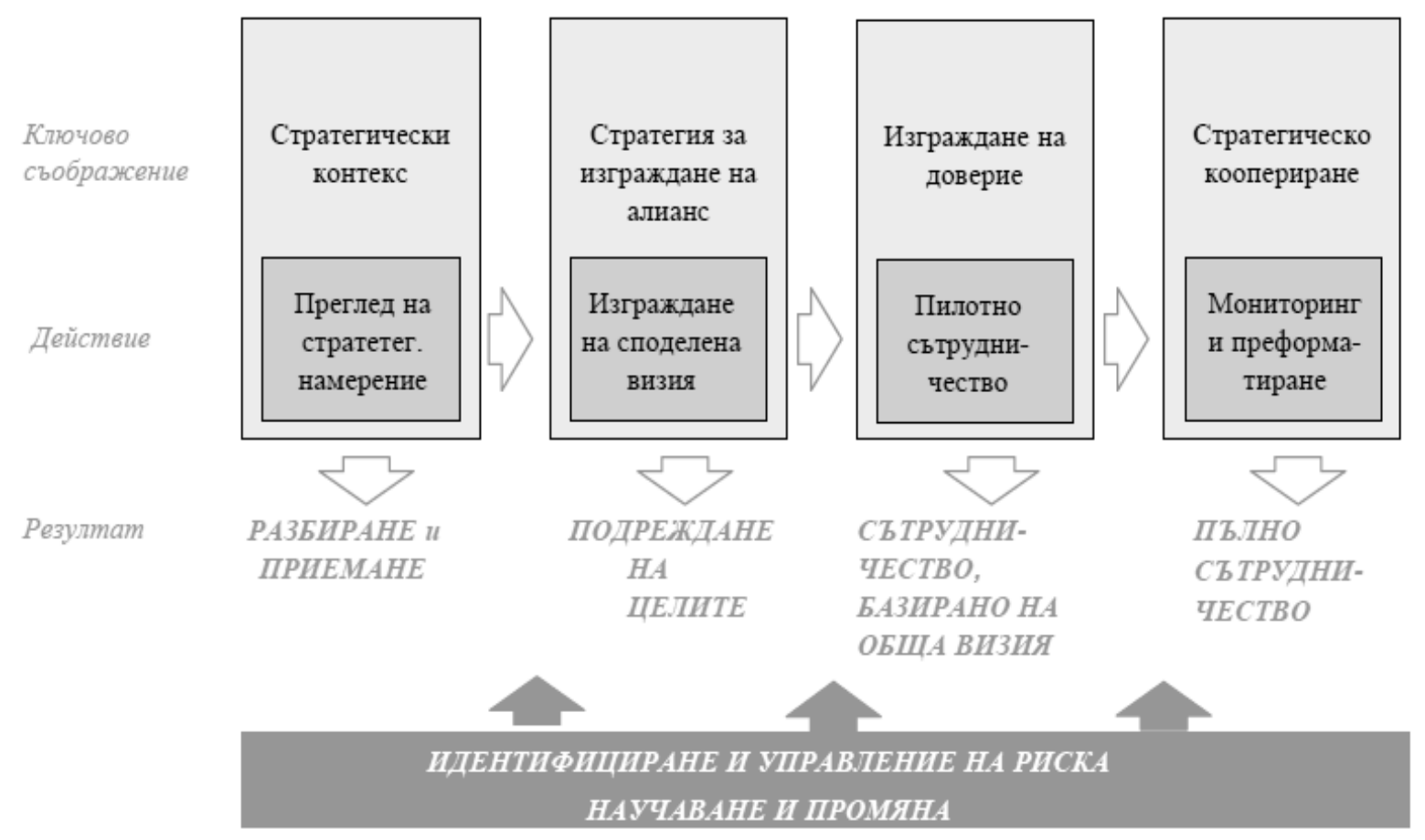

Фигура 3. Модел за създаване на стратегически алианси (Seppälä, 2004, p.160) 
Второто ключово съображение всъщност е първата стъпка в процеса на формиране на алианса. От важно значение в този етап е наличието на ясна алиансна стратегия, която да е обвързана с целите на алианса и със стратегическото намерение на организацията. Действието тук е насочено към изграждане на споделена визия за сътрудничество, което включва опознаване и разбиране на бизнеса, структурата, стратегическите намерения и стратегията на алианса от страна на потенциалния партньор. Моделът допуска, че единствено ако е създадена достатъчно здрава основа от споделената визия, може да се продължи към следващия етап от процеса на създаване на стратегическия алианс.

Централно място в създаването на партньорства заема доверието, наличието на което е важна предпоставка за успеха на стратегическия алианс, но то подлежи на трудно измерване и определяне (Demirbag et al., 2002). В предложения от Сепала модел се допуска, че третото ключово съображение по създаване на доверие всъщност започва с изграждането на споделена визия за сътрудничество. За разлика от съществуващите модели, които акцентират върху договарянето между страните след като са поставени общите цели (Contractor et al, 2000; Campbell et al.,2001), авторьт тук предвижда т.нар. пилотно сътрудничество, което има за цел да подпомогне изграждането на доверие и да осигури допълнителна възможност за опознаване между страните. Основата на доверието се гради както върху споделената визия, така и върху социални взаимоотношения, породени от съвместната дейност. Именно това ограничено сътрудничество в определена област може да се използва за тестване на потенциала за по-нататъшно тясно коопериране и като средство за ограничаване на последващи конфликти между страните (Young et al, 1989).

Последното ключово съображение в модела е свързано с факта, че стратегическият алианс не е еднократна сделка и е необходимо неговото постоянно наблюдение и пренастройване в унисон с промените, които настъпват в организациите и в стратегическия контекст (Doz et al., 1998). За разлика от изследването на Доз, в което се акцентира върху еволюцията на алианса след като бъде формално създаден, то в посочения модел на Сепала се прогнозира и проследява неговото развитие още в подготвителния период, преди фактическото му учредяване. Следвайки модела, организациите се ангажират в създаването на споделена визия, изграждане на доверие и осъществяване на пилотно сътрудничество, като участват в единен процес на взаимно опознаване, преоценка на ситуацията и оптимално пренастройване съобразно новите условия (Seppälä, 2004).

Въз основа на анализа на различни авторови виждания за същността, мотивите и процеса на създаване на стратегически алианси считаме, че модела на Н. Евънс, представящ стратегическия процес на коопериране, в достатьчно пълна степен разкрива логиката на формирането на стратегически партньорства.

Модельт използва методологията на стратегическото управление и включва три основни компонента:

- стратегически анализ;

- формулиране на стратегия;

- реализиране на стратегията.

Взаимовръзките между тях са илюстрирани по-долу (фиг. 4):

По отношение на първия компонент на модела следва да посочим, че стратегическият анализ се свежда до анализ на вътрешната и външната среда и извеждане на движещите сили на промяната в организацията.

При формулиране на стратегията са налице три възможни алтернативи:

- самостоятелно развитие (вътрешен растеж);

- коопериране (съвместна дейност);

- сливания и погльщания.

При самостоятелното развитие организацията използва вътрешните си ресурси за реализиране на стратегията си за развитие. Кооперирането (стратегическия алианс) 
предполага споделяне на ресурси и съвместна дейност с друга организация. Сливането и поглъщането е свързано със загуба на автономност и създаването на нова организация.
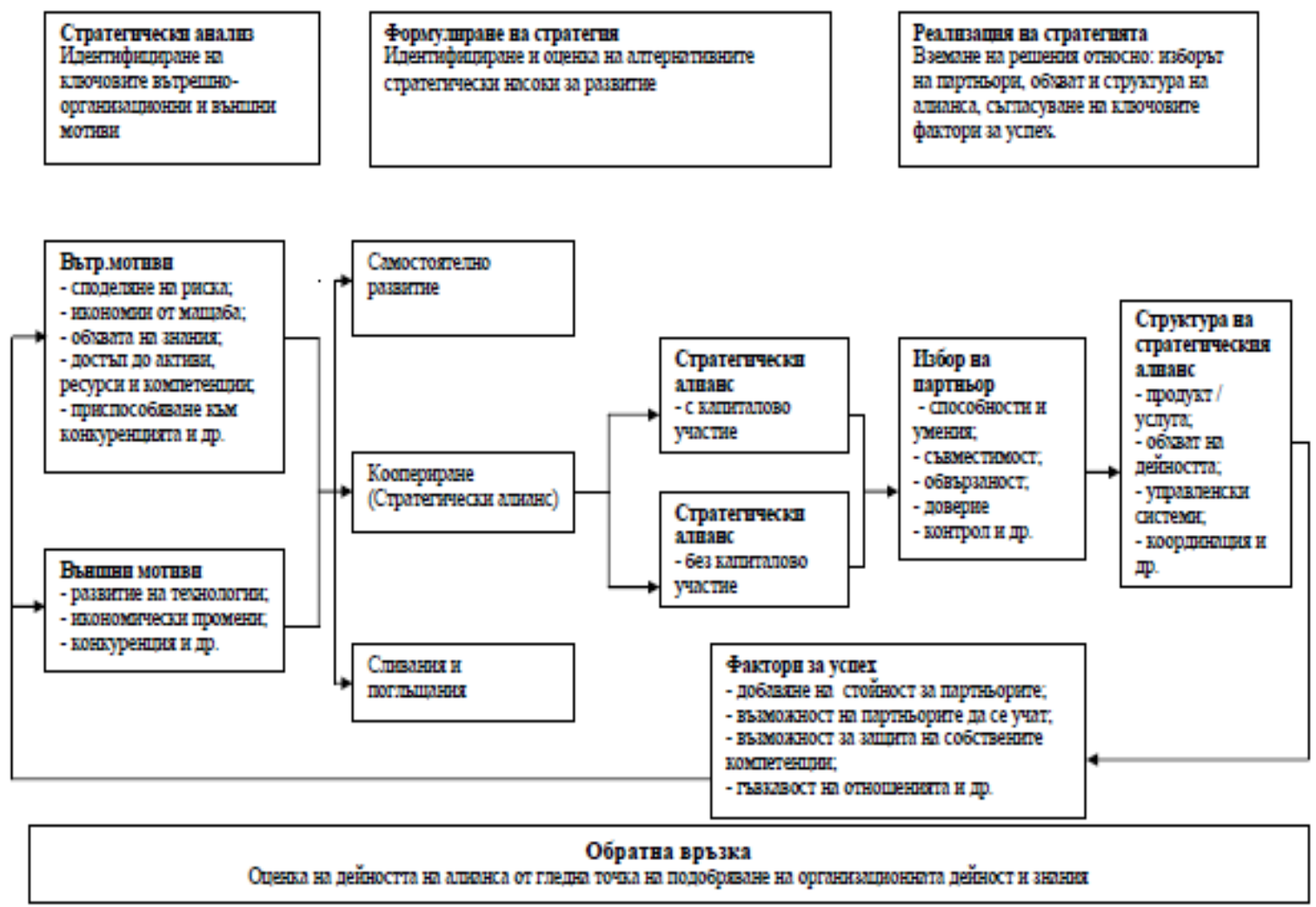

Фигура 4. Модел на стратегическия процес на коопериране (Evans, 2001, p.233)

От своя страна, кооперирането може да се осъществи по два начина:

- Стратегически алианс с капиталово участие;

- Стратегически алианс без капиталово участие.

Третият компонент на модела - реализация на стратегията - предполага самото изграждане на стратегическия алианс и е свързан със следните дейности:

- избор на партньор въз основа на предварително определени критерии за оценка;

- изграждане структурата на стратегическия алианс от гледна точка на неговия продукт/услуга, обхвата на съвместната дейност, управленски системи, координация и др;

- определяне на ключовите фактори за успех на алианса.

Обратна връзка - оценката за алианса се прави от гледна точка на усъвършенстването на дейността на организацията и натрупаните знания.

\section{2. Модел за формиране на стратегически алианси в болничния сектор}

В разработването на модела за създаване на стратегически алианси в болничния сектор сме имали предвид следните ограничения:

- Използвана е логиката на стратегическия подход и методологията на стратегическото управление. По своята същност стратегическият алианс е средство за реализиране на стратегията на развитие на болницата;

- Като основа е използван модел на стратегическия процес на коопериране, който е адаптиран спрямо спецификите на здравеопазването и в частност, на болничния сектор.

- Дефинирани са онези етапи и дейности, които се включват в процеса на 
създаване на стратегически алианси и могат да бъдат използвани за целите на модела;

- Някои от елементите в модела са специфични и произтичат от характеристиките на здравната система в България.

При тези условия целта на предлагания модел е да се разкрият eтапите $u$ механизмите за създаване на успешен стратегически алианс с участието на болница.

Принципите, на които се основава предложения модел са:

- системност и непрекъснатост на етапите;

- равнопоставеност на оперативните и стратегическите решения;

- ясно и точно определени критерии, подходи, фактори и показатели;

- обективност при избора на фактори и показатели от средата;

- управляемост на процеса чрез различни механизми;

- отговорност за избора на стратегически решения;

- прозрачност и яснота на приложение на механизмите на модела;

- всеобхватност и простота по отношение на приложимостта му;

- отвореност на процедурите, която позволява добавянето на нови елементи, съобразно специфичните особености на здравната система, в която функционира лечебното заведение за болнична помощ.

Като предпоставки за прилагане на модела бихме могли да посочим следните:

- създаването на успешен стратегически алианс с участието на болница предполага наличието на осъзната необходимост от страна на висшите мениджъри и желание за сътрудничество с други организации;

- приложението на модела е ограничено от действието на механизмите на държавно регулиране в областта на здравеопазването;

- прилагането на модела изисква последователност в реализирането на етапите и дейностите в него;

- необходимост от знания и умения на специалистите за анализ, оценка и вземане на стратегически решения;

- познаване на методиката за приложението му;

- д достоверност на информацията, която се използва като първична;

- наличие на необходимата информация за болницата и за външната среда;

\section{Основните постановки в модела са:}

- Може да се разглежда като общ, валиден за всички болници;

- Информацията, използвана в модела, трябва да се актуализира на определени кратки периоди от време, които зависят от динамиката на процесите във външната и вътрешната среда. Причината е в необходимостта от бърза адаптация към промените.

- Модельт използва резултативна информация от статистически, социологически, маркетингови и други изследвания както фактори и показатели за анализ, оценка и сравнение.

- В модела се използват методите на анализ и синтез, системният анализ, стратегическият анализ, сравнително-оценъчният подход и експертната оценка

В предложения модел за създаване на стратегически алианси в болничния сектор са включени следните елементи:

- Външна среда на болницата и външни мотиви за сътрудничество;

- Вътрешна среда и вътрешни мотиви за сътрудничество;

- Интереси на стейкхолдерите;

- Закон за лечебните заведения;

- Закон за здравето; 
- Търговски закон;

- Стратегическо решение за СА;

- Критични фактори за успех;

- Собственик (принципал);

- орма на стратегическия алианс;

- Неформално сътрудничество;

- Договорни споразумения;

- Капиталово участие;

- Партньор;

- Лечебно заведение;

- Здравно заведение;

- Друга организация извън здравната система;

- Стратегически алианс;

- Организационна структура;

- Цели;

- Правилници;

- Договори;

- Съвместни управленски системи;

- Информационна система;

- Мотивация на персонала;

- Контрол и оценка;

- Резултати и обратна връзка.

Логиката на връзките и зависимостите между елементите в модела е представена на фигура 5.

Информационното осигуряване на модела е резултат от системно проучване на информация за факторите и определящите ги показатели за средата.

За приложение на разработения модел за създаване на стратегически алианси в болничния сектор извеждаме методика, която включва следните етапи:

А. Вземане на решение за създаване на стратегически алианс.

А1. Анализ на външната среда на болницата.

А2. Анализ и оценка на вътрешната среда и стратегическите цели.

А3. Анализ на целесъобразността от създаването на стратегически алианс.

Б. Избор на подходяща организационна форма на стратегическия алианс.

Б1. Определяне на критерии за избор на подходяща форма.

Б2. Анализ на възможните организационни форми.

Б3. Избор на организационна форма.

В. Избор на партньор на болницата в стратегическия алианс.

В1. Определяне на потенциални партньори.

В2. Дефиниране на ограничителни условия.

В3. Определяне на критерии за избор.

B4. Анализ и оценка на потенциалните партньори.

B5. Избор на партньор.

Г. Изграждане на стратегическия алианс.

Г1. Проектиране на организационна структура.

Г2. Дефиниране на параметрите на съвместната дейност.

Г3. Договаряне между страните.

Г4. Създаване на механизъм за изграждане и поддържане на доверие между

страните.

Г5. Създаване на механизъм за разрешаване на конфликти.

Г6. Механизъм за контрол и оценка на изпьлнение. 




Фигура 5. Модел за създаване на стратегически алианси в болничния сектор

\section{Заключение}

Изводите и обобщенията от представения модел за създаване на стратегически алианси в болничния сектор са следните:

Първо, предложеният модел интегрира стратегическия процес на коопериране със спецификите на управлението на лечебните заведения за болнична помощ. Акцентьт в неговото приложение е създаването на успешен стратегически алианс.

Второ, разработеният модел има съставни елементи и се подчинява на определени принципи и изходни постановки. При тези условия целта му е да се разкрият етапите $u$ механизмите за създаване на успешен стратегически алианс от болницата. 
Трето, елементите в модела са свързани посредством определени действия, обособяващи етапи в единна технология на процеса на формиране на алианси и неговите стратегически аспекти. Тя ни формира методиката за приложение на предложения модел.

\section{References}

1. Brouther, K., Brouthers, D., Harris, P. (1997) The Five Stages of the Co-operative Venture Strategy Process. Journal of General Management. 23 (1). pp. 39-52.

2. Buchel, B. (2000) Framework of Joint Venture Development: Theory-Building Through Qualitative Research. Journal of Management Studies. 37(5). pp. 637-661.

3. Campbell, E., Reuer, J. (2001) International Alliance Negotiations: Legal Issues for General Managers. Business Horizons. 44 (1). pp. 19-26.

4. Contractor, F., Ra, W. (2000). Negotiating alliance contracts: Strategy and behavioral effects of alternative compensation arrangements. International Business Review. 9 (3). pp. 271-299.

5. Daz, T., Teng, B. (1997) Sustaining Strategic Alliances: Options and Guidelines. Journal of General Management. 22 (4). pp. 49-63.

6. Demirbag, M., Weir, D., Mirza, H. (2002) Trust, Inter-partner Conflicts, Cultural Distance, Commitment and Joint Venture Performance: An Empirical Analysis of International Joint Ventures in Turkey. Journal of Transnational Management Development. 8 (1). pp. 111-140.

7. Doz, Y., Hamel, G. (1998) Alliance Advantage - The Art of Creating Value Through Partnering. Boston, MA: Harvard Business School Press.

8. Doz, Y. (1996) The Evolution of Cooperation in Strategic Alliances: Initial Conditions or Learning Process. Strategic Management Journal. 17. pp. 55-83.

9. Evans, N. (2001) Collaborative strategy: an analysis of the changing world of international airline alliances. Tourism Management. 3 (22). pp. 229-243.

10. Hoffman, W., Schlosser, R. (2001) Success factors for Strategic Alliances in Small and Medium-sized Enterprises - An Empirical Survey. Long Range Planning. 34 (3). pp. 357-381.

11. Kinderis, R., Jucevicius, G. (2013) Strategic Alliances - Their Definition and Formation. Latgale National Economy Research. 1(5). pp. 106-128.

12. Lewis J.D. (1990) Partnerships for profit. Structuring and managing strategic alliances. New York: The Free Press, Macmillan Inc.

13. Mitev, B. (2005) Protsesat na formirane na strategicheski aliansi v konteksta na Evropeyskata perspektiva pred balgarskiya biznes. Nauchni trudove. RU „Angel Kanchev”. 44 (5.1). pp. 7781.

14. Mitev, B., Rohova, M. (2006) Strategicheskite aliansi v zdraveopazvaneto. Savremenni upravlenski praktiki IV. Burgas. 1. pp. 211-221.

15. Mitsuhashi, H. (2002) Uncertainty in selecting alliance partners; The three reduction mechanisms and alliance formation process. The International Journal of Organizational Analysis. 10 (2). pp. 109-133.

16. Ring, P., Van de Ven, A. (1994) Developmental Processes of Cooperative Interorganizational Relationships. Academy of Management Review. 19 (1). pp. 90-118.

17. Seppälä, M. (2004) Model for Creating Strategic Alliances. A Study of Inter-firm Cooperation in the North European ICT Sector. (Doctoral dissertation. Finland).

18. Whipple, J.S., Frankel, R. (1998) The Alliance Formation Process. International Food and Agribusiness Management Review. 1 (3). pp. 335-357.

19. Young, L., Wilkinson, I. (1989) The Role of Trust and Cooperation in Marketing Channels: a Preliminary Study. European Journal of Marketing. 23 (2). pp. 109-122.

20. Zafirova, Tsv., Mitev, B. (2007) Podgotovka za strategichesko reshenie po sazdavane na aliansi v bolnichniya sektor. Savremenni upravlenski praktiki V, Burgas Free University. 2. pp. 189205. 